\title{
Detailed Theoretical Model for Adjustable Gain-Clamped Semiconductor Optical Amplifier
}

\author{
Lin Liu, ${ }^{1}$ Craig Michie, ${ }^{1}$ Anthony E. Kelly, ${ }^{2}$ and Ivan Andonovic ${ }^{1}$ \\ ${ }^{1}$ Department of Electronic and Electrical Engineering, University of Strathclyde, Glasgow G1 1XW, UK \\ ${ }^{2}$ Department of Electronics and Electrical Engineering, University of Glasgow, Glasgow G12 8LT, UK
}

Correspondence should be addressed to Ivan Andonovic, i.andonovic@eee.strath.ac.uk

Received 1 October 2011; Revised 7 February 2012; Accepted 11 February 2012

Academic Editor: Luciano Mescia

Copyright (C) 2012 Lin Liu et al. This is an open access article distributed under the Creative Commons Attribution License, which permits unrestricted use, distribution, and reproduction in any medium, provided the original work is properly cited.

\begin{abstract}
The adjustable gain-clamped semiconductor optical amplifier (AGC-SOA) uses two SOAs in a ring-cavity topology: one to amplify the signal and the other to control the gain. The device was designed to maximize the output saturated power while adjusting gain to regulate power differences between packets without loss of linearity. This type of subsystem can be used for power equalisation and linear amplification in packet-based dynamic systems such as passive optical networks (PONs). A detailed theoretical model is presented in this paper to simulate the operation of the AGC-SOA, which gives a better understanding of the underlying gain clamping mechanics. Simulations and comparisons with steady-state and dynamic gain modulation experimental performance are given which validate the model.
\end{abstract}

\section{Introduction}

Semiconductor optical amplifiers (SOAs) have attracted considerable attention during the last two decades, for use in evolving optical communication networks. SOAs can be used as not only optical amplifiers, but also signal processing devices such as wavelength converters [1], optical switches [2], and electro-optical mixers [3]. In terms of optical amplification, the key issue of operating SOAs is the management of the input optical signal power, which must be maintained within the linear regime of operation; otherwise the device would be driven into saturation causing unwanted intersymbol interference (ISI) or patterning. In order to solve this implementation problem, many different types of gainclamped semiconductor optical amplifiers (GC-SOAs) have been proposed $[4,5]$. Recently, an adjustable gain-clamped semiconductor optical amplifier (AGC-SOA) designed to maximize the output saturated power at a clamped gain which can be adjusted was reported [6].

\section{AGC-SOA}

The AGC-SOA is a semiconductor optical amplifier topology which has the unique capability to provide variable gain and maintain linear operation through gain clamping over a wide ( $40 \mathrm{~dB})$ dynamic range, without compromising the saturable output power of the device [6]. A key advantage of this approach is that there are no mechanical tuning elements, and hence the gain can be adjusted via direct electrical control at ns timescales. While the operation of this device has been presented previously for the static gain case [6], and its behaviour under dynamic gain modulation conditions [7], the underlying mechanics is not well understood. Here, a theoretical model for an AGC-SOA has been established, based on the wideband steady-state numerical model of a SOA [8]. The travelling amplified spontaneous emission (ASE) power and spectrum within a ring cavity, steady-state, and dynamic gain modulation have been studied. Simulations are in broad agreement with experiment results.

Figure 1 illustrates conceptually the design of the adjustable gain clamped SOA (AGC-SOA). The architecture comprises two active (gain) regions defining a data path through the signal SOA (SOA1) and a laser cavity containing SOA1 and a control SOA (SOA2). SOA1 amplifies light in the signal path. The lasing mode derives gain from both SOA1 and SOA2. The composite gain provided by both SOAs regulates the condition for the onset of lasing. This in turn defines the carrier concentration (gain) of the signal SOA. Hence, 


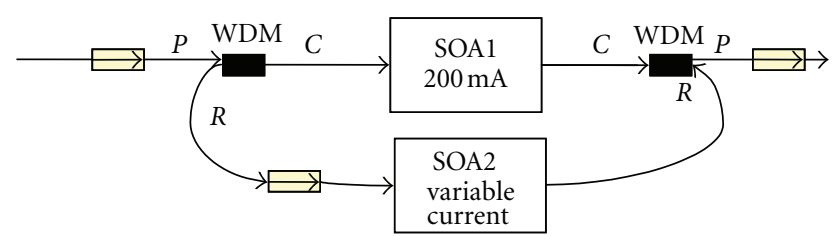

FIGURE 1: Counterpropagating ring laser adjustable gain-clamped SOA (AGC-SOA) implementation.

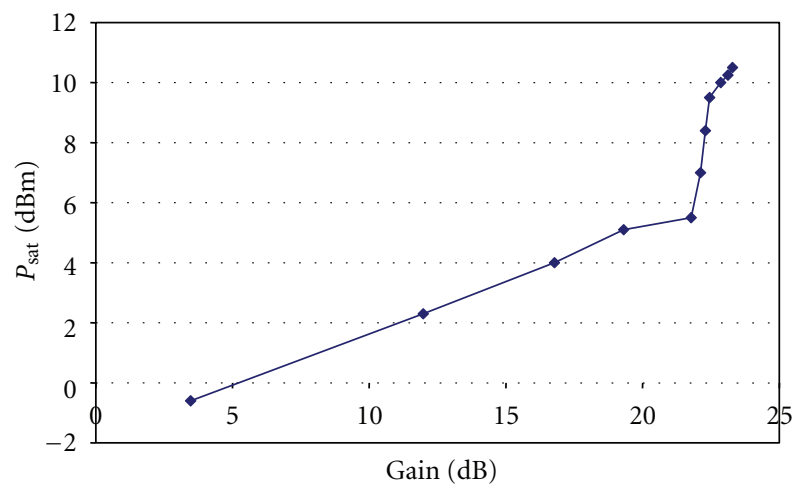

FIgURE 2: $P_{\text {sat }}(\mathrm{dBm})$ variation as a function of SOA gain $(\mathrm{dB})$.

by controlling the drive to SOA2, the gain imparted by SOA 1 can be adjusted. SOA1 is continually operated at full current, and therefore, the AGC-SOA allows signals to be amplified by SOA1 at a clamped gain which is varied by SOA2. This maximises the saturation output power thereby maintaining an extended linear regime [6].

The key advantage that the AGC-SOA offers over other optical amplifiers is that it enables the gain to be adjusted directly through the drive current to the clamping SOA without the dramatic loss of $P_{\text {sat }}$. Hence, linear operation is maintained over a wider range of input signals. In standard SOAs, it is possible to adjust the gain by altering the drive current however, as is demonstrated in the experimental measurement shown in Figure 2, and this leads to a dramatic loss in $P_{\text {sat }}$.

At high gains, where the SOA is highly inverted, the $P_{\text {sat }}$ value is at its highest. However, in this region, adjusting the small signal gain through bias current has a dramatic effect on the $P_{\text {sat }}$ value. In the example depicted in Figure 2, at high gains the $P_{\text {sat }}$ changes with gain at a rate of $\sim 3 \mathrm{dBm} / \mathrm{dB}$ i.e., for every $\mathrm{dB}$ that the gain is reduced, the $P_{\text {sat }}$ value drops by $3 \mathrm{dBm}$. As the drive current is further reduced, the drop in $P_{\text {sat }}$ with gain is weaker at $\sim 0.4 \mathrm{dBm} / \mathrm{dB}$. However, by the time that this point has been reached, the $P_{\text {sat }}$ value is already significantly compromised ( $5 \mathrm{dBm}$ compared to the high gain value of $10 \mathrm{dBm}$ ). The AGC-SOA enables gain modulation to be achieved without this dramatic loss of $P_{\text {sat }}$ value.

Figure 3 depicts the gain of AGC-SOA as a function of output power for a set of different clamping currents ranging from $0 \mathrm{~mA}$ to $200 \mathrm{~mA}$. The $P_{\text {sat }}$ values are constant over the range of clamping currents despite significant gain reduction $(>20 \mathrm{~dB})$, demonstrated in Figure 4.

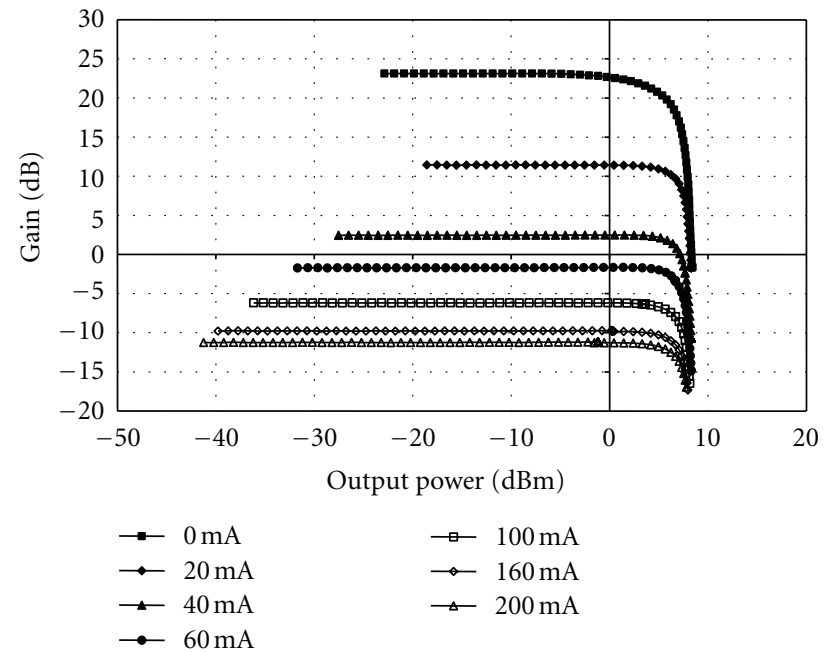

FIGURE 3: AGC-SOA gain as a function of output power at different clamping currents. Legend: bias current of SOA2.

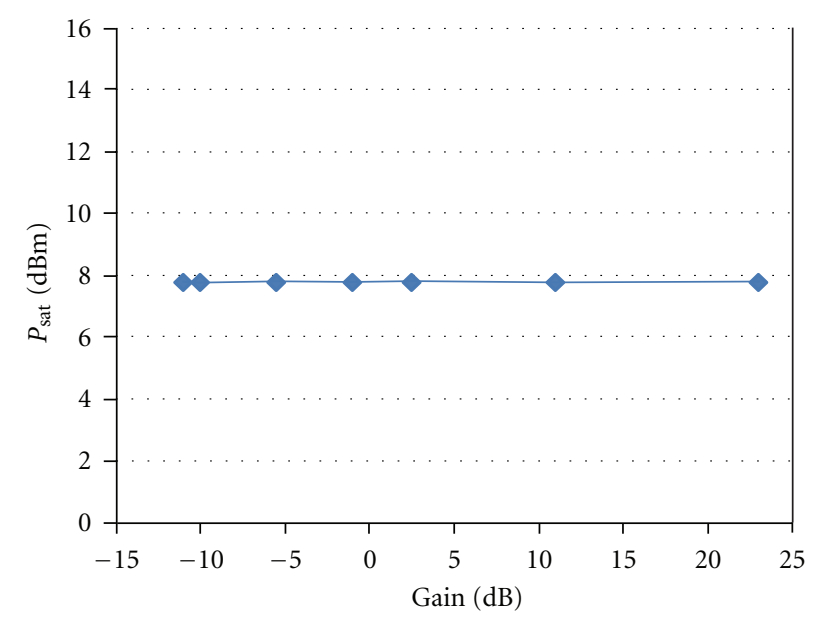

Figure 4: $P_{\text {sat }}(\mathrm{dBm})$ variation as a function of AGC-SOA gain $(\mathrm{dB})$.

Additional steady-state experimental results such as gain, Noise Figure (NF), and $P_{\text {sat }}$ value were demonstrated in [6]. The dynamic gain modulation performance of AGC-SOA was further studied in [7]. In a dynamic packet equalisation scenario, the AGC-SOA is able to adjust and stabilise its gain in less than $2 \mathrm{~ns}$, within the period of the guard band of the passive optical network (PON) transmission at $1.25 \mathrm{Gbit} / \mathrm{s}$ on the upstream direction, 32 bit periodsis equates to $26 \mathrm{~ns}$. Similarly for 10-Gigabit-capable, PON system, the guard time is 64 bits at $2.5 \mathrm{Gbit} / \mathrm{s}$ on the upstream direction [9], this equates to $26 \mathrm{~ns}$ [9].

\section{Model of AGC-SOA}

Several numerical models have been developed to investigate the characteristics of both conventional SOAs [8] and gainclamped SOAs using either an external $[10,11]$ or internal $[12,13]$ lasing mode. However, the underlying mechanism of gain clamping achieved by adjusting wideband amplified 
TABLe 1: Device parameters used in AGC-SOA simulation.

\begin{tabular}{llcc}
\hline Symbol & Parameters & SOA1 & SOA2 \\
\hline$L_{c}$ & Central active region length & $400 \mu \mathrm{m}$ & $840 \mu \mathrm{m}$ \\
$L_{t}$ & Tapered active region length & $200 \mu \mathrm{m}$ & $160 \mu \mathrm{m}$ \\
$d$ & Active region thickness & $0.4 \mu \mathrm{m}$ & $0.1 \mu \mathrm{m}$ \\
$W$ & Active region width & $0.4 \mu \mathrm{m}$ & $1.1 \mu \mathrm{m}$ \\
$y$ & Molar fraction of arsenide & 0.892 & 0.816 \\
$\Gamma$ & Confinement factor & $5 \times 10^{-5}$ & 0.15 \\
$R_{1}$ & Input facet reflectivity & $5 \times 10^{-5}$ & $1 \times 10^{-5}$ \\
$R_{2}$ & Output facet reflectivity & $3 \mathrm{~dB}$ & $1 \times 10^{-5}$ \\
$\eta_{\text {in }}$ & Input coupling loss & $3 \mathrm{~dB}$ & $2 \mathrm{~dB}$ \\
$\eta_{\text {out }}$ & Output coupling loss & $6200 \mathrm{~m}^{-1}$ & $2 \mathrm{~dB}$ \\
$K_{0}$ & Carrier-independent absorption loss coefficient & $7.5 \times 10^{-21} \mathrm{~m}^{2}$ & $3000 \mathrm{~m}^{-1}$ \\
$K_{1}$ & Carrier-dependent absorption loss coefficient & 3.22 & $6.83 \times 10^{-21} \mathrm{~m}^{2}$ \\
$n_{1}$ & Active region refractive index & 3.56 \\
$C_{1}$ & & & $3.7 \times 10^{8} \mathrm{~s}^{-1}$ \\
$C_{2}$ & Recombination coefficients & $5.6 \times 10^{-16} \mathrm{~m}^{3} / \mathrm{s}$ \\
$C_{3}$ & & $3 \times 10^{-41} \mathrm{~m}^{6} / \mathrm{s}$ \\
$\alpha_{\text {coupler }}$ & WDM coupler pass band insertion loss & $0.5 \mathrm{~dB}^{2}$ \\
\hline
\end{tabular}

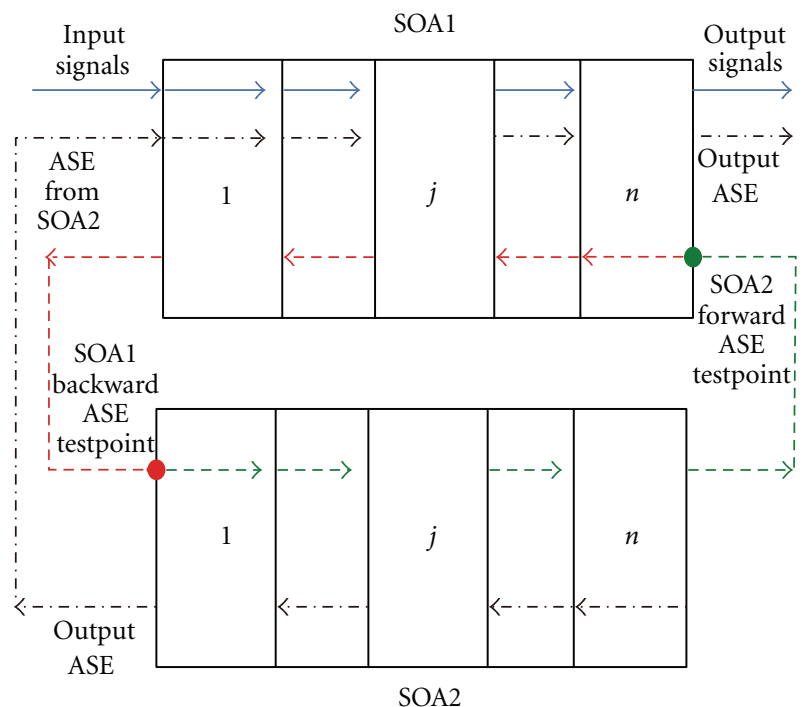

FIGURE 5: Schematic of the simulation model for an AGC-SOA.

spontaneous emission (ASE) power still remains largely unknown. In this study, the wideband steady-state SOA model [8] is adapted to form a ring cavity; the evolution of travelling ASE power and spectrum within the ring cavity, important for gain clamping, is then characterised. The gain, Noise Figure (NF), and maximum output power at gain saturation $\left(P_{\text {sat }}\right)$ of an AGC-SOA under different clamping currents are also studied. Based on this model, the timescale for adjusting and stabilising the gain, crucial for dynamic packet equalisation, is evaluated.

As shown in Figure 5, ASE circulating within the AGCSOA travels in clockwise and counterclockwise directions, however, the isolator in the ring cavity ensures that ASE travelling in the clockwise direction is not amplified. Thus, the counterclockwise ASE generated by both SOAs accounts for gain clamping. In this model, the two SOAs are simulated as independent modules using different sets of material parameters summarised in Table 1. (Typical bulk SOA parameters from $[8,14,15])$. Both SOAs generate ASE in the forward and backward directions in the active regions. In each SOA, the ASE profile extends over $1300 \mathrm{~nm} \sim 1650 \mathrm{~nm}$ and is partitioned into discrete frequency bands.

In order to simulate the wideband ASE profile for a single SOA, the material gain coefficient of the SOA active region in an InGaAsP direct bandgap bulk material is given by $[8,16]$

$$
\begin{aligned}
g_{m}(\nu, n)= & \frac{c^{2}}{4 \sqrt{2} \pi^{3 / 2} n_{1}^{2} \tau \nu^{2}}\left(\frac{2 m_{e} m_{\mathrm{hh}}}{\hbar\left(m_{e}+m_{\mathrm{hh}}\right)}\right)^{3 / 2} \\
& \times \int_{0}^{\infty}\left(v^{\prime}-\frac{E_{g}}{h}\right)^{1 / 2} \times\left(f_{c}\left(\nu^{\prime}\right)-f_{v}\left(\nu^{\prime}\right)\right) \\
& \times\left(\frac{2 T_{0}}{1+\left(\nu^{\prime}-v\right)^{2}\left(2 \pi T_{0}\right)^{2}}\right) d \nu^{\prime} \\
= & \frac{c^{2}}{4 \sqrt{2} \pi^{3 / 2} n_{1}^{2} \tau \nu^{2}}\left(\frac{2 m_{e} m_{\mathrm{hh}}}{\hbar\left(m_{e}+m_{\mathrm{hh}}\right)}\right)^{3 / 2} \\
& \times\left(v-\frac{E_{g}}{h}\right)^{1 / 2} \times\left(f_{c}(v)-f_{v}(\nu)\right),
\end{aligned}
$$

where $c$ is the velocity of light in vacuum, $v$ is the optical frequency, $n_{1}$ is the active region refractive index, $\tau$ is the radiative carrier recombination lifetime, $\hbar$ is the normalized Planck constant, $m_{e}$ is the effective mass of an electron in conduction band (CB), $m_{\mathrm{hh}}$ is the effective mass of a heavy hole in valence band $(\mathrm{VB}), E_{g}$ is the band gap energy of the material, $T_{0}$ is the mean lifetime for the coherent interaction of electrons with a monochromatic field, and $f_{c}$ and $f_{v}$ are the Fermi-Dirac distributions for the conduction and valence bands, respectively. 
The gain coefficient can be divided into two parts: stimulated emission rate $R_{e}$ and stimulated absorption rate $R_{a}$,

$$
g_{m}(\nu, n)=R_{e}(\nu, n)-R_{a}(\nu, n),
$$

where

$$
\begin{aligned}
R_{e}(\nu, n)= & \frac{c^{2}}{4 \sqrt{2} \pi^{3 / 2} n_{1}^{2} \tau \nu^{2}}\left(\frac{2 m_{e} m_{\mathrm{hh}}}{\hbar\left(m_{e}+m_{\mathrm{hh}}\right)}\right)^{3 / 2} \\
& \times\left(\nu-\frac{E_{g}}{h}\right)^{1 / 2} \times f_{c}(\nu)\left(1-f_{v}(\nu)\right), \\
R_{a}(\nu, n)= & \frac{c^{2}}{4 \sqrt{2} \pi^{3 / 2} n_{1}^{2} \tau \nu^{2}}\left(\frac{2 m_{e} m_{\mathrm{hh}}}{\hbar\left(m_{e}+m_{\mathrm{hh}}\right)}\right)^{3 / 2} \\
& \times\left(\nu-\frac{E_{g}}{h}\right)^{1 / 2} \times f_{v}(\nu)\left(1-f_{c}(\nu)\right) .
\end{aligned}
$$

The propagation of optical field in the SOA can be described as

$$
\begin{aligned}
& \frac{d A_{j}^{+}}{d z}=\left[-i \beta+\frac{1}{2}\left(\Gamma g_{m}(\nu, n)-\alpha\right)\right] A_{j}^{+}, \\
& \frac{d A_{j}^{-}}{d z}=\left[i \beta-\frac{1}{2}\left(\Gamma g_{m}(\nu, n)-\alpha\right)\right] A_{j}^{-},
\end{aligned}
$$

where $A_{j}^{+}$and $A_{j}^{-}$are the forward and backward travelling waves of the $j$ th active region section, respectively, $\beta$ is the propagation constant, $\Gamma$ is the confinement factor, and $\alpha$ is the internal loss coefficient.

The propagation of the spontaneous emission field is presented as

$$
\begin{aligned}
\frac{d S_{k}^{+}}{d z} & =\left[\Gamma g_{m}\left(\nu_{k}, n\right)-\alpha\right] S_{k}^{+}+R_{\mathrm{sp}}\left(v_{k}, n\right), \\
\frac{d S_{k}^{-}}{d z} & =-\left[\Gamma g_{m}\left(\nu_{k}, n\right)-\alpha\right] S_{k}^{-}-R_{\mathrm{sp}}\left(v_{k}, n\right) .
\end{aligned}
$$

$S_{k}^{+}$and $S_{k}^{-}$are the forward and backward amplified spontaneously emitted photon densities per unit frequency spacing centred at frequency $v_{k} . R_{\mathrm{sp}}\left(v_{k}, n\right)$ is the ASE noise coupled into $S_{k}^{+}$or $S_{k}^{-}$, and it is expressed as $R_{\mathrm{sp}}\left(\nu_{k}, n\right)=$ $\Gamma R_{e}\left(\nu_{k}, n\right) \Delta v, \Delta \nu$ is frequency spacing.

The carrier rate equation is presented by

$$
\begin{aligned}
\frac{d n(Z)}{d t}= & \frac{I}{q V}-R(n(z))-\sum_{j=1}^{n} \frac{\Gamma g_{m}(\nu, n)}{A_{\text {cross }}}\left(A_{j}^{+}+A_{j}^{-}\right) \\
& -\sum_{k=1}^{m} \frac{2 \Gamma g_{m}\left(\nu_{k}, n\right)}{A_{\text {cross }}}\left(S_{k}^{+}+S_{k}^{-}\right) .
\end{aligned}
$$

The recombination rate term $R(n(z))$ is given by

$$
R(n)=C_{1} n+C_{2} n^{2}+C_{3} n^{3},
$$

where $C_{1}, C_{2}$, and $C_{3}$ are the nonradiative recombination, bimolecular radiative recombination, and auger recombination coefficients, respectively.

The numerical model for the whole system of Figure 5 is achieved using iterative circulations. In the first iteration, the ASE in both directions of SOA2 is calculated assuming no

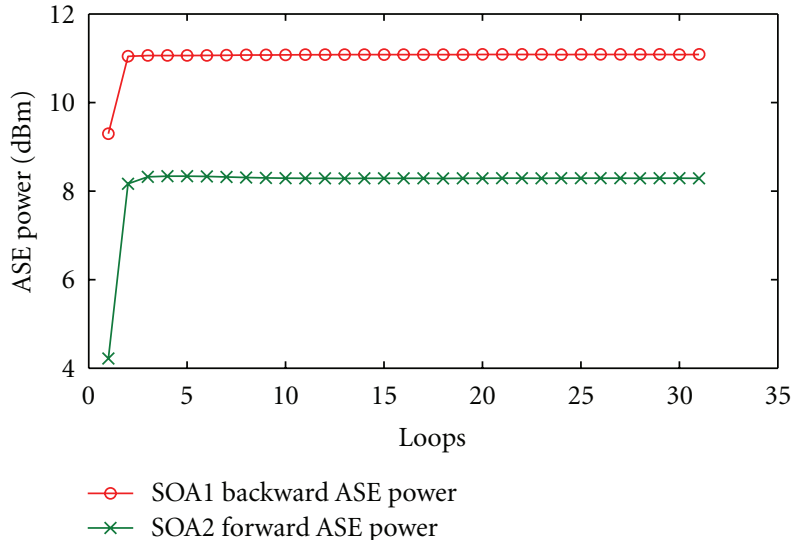

FIGURE 6: Counterclockwise ASE power after $N$ loops. Cross: SOA2 output ASE power. Circle: SOA1 output ASE power.

ASE power is coupled in. Then the ASE generated by SOA2 travels in both clockwise and counterclockwise order towards SOA1. Under this boundary condition, the ASE originated from SOA2 together with the one generated by SOA1 is amplified by SOA1 as it travels through; however, only the backward ASE inside the SOA1 is input to SOA2. For any successive iterations, the ASE from SOA1 couples into SOA2 before SOA2 generates ASE. When ASE travels inside the ring cavity, the facet reflectivity and coupling loss of both SOAs, the insertion loss for the isolator, and WDM couples are taken into account. The round trip time is $\sim 1.67$ ns namely, the fibre length is about $0.5 \mathrm{~m}$. Therefore, fibre loss and dispersion are neglected. The iterative procedure is terminated when the maximum difference of the ASE powers at each discrete frequency band between successive iterations is less than the desired tolerance. The numerical model is implemented using Matlab.

\section{Simulation Results and Discussions}

The characteristics of AGC-SOA were studied from the initial state when no input signal is introduced. The ASE power after every counterclockwise ASE round trip is recorded. In Figure 6, the counterclockwise output ASE powers from SOA2 and SOA1 are displayed after each loop transit.

The ASE power increases rapidly within the first 3 loops and then stabilises. Since the cavity round trip time is $\sim 1.7 \mathrm{~ns}$, stabilising the travelling ASE power in the loop takes between 1.7 and $5.1 \mathrm{~ns}$ (several round trips). The ASE spectrum within the clamping mode was examined after every circulation. Figure 7 shows the SOA2 output ASE spectrum at different loop transits. In the first loop, the output ASE power from SOA2 is relatively low, and the whole spectrum is divided into two parts falling outside the C-band due to presence of the WDM coupler in the ring cavity.

Initially, the ASE power within the S-band is greater than that within the L-band. However, as the lasing mode becomes established, the output ASE power develops as predicted in Figure 6. With the ASE power within the S-band decreasing significantly, the spectrum becomes sharp and narrow. 


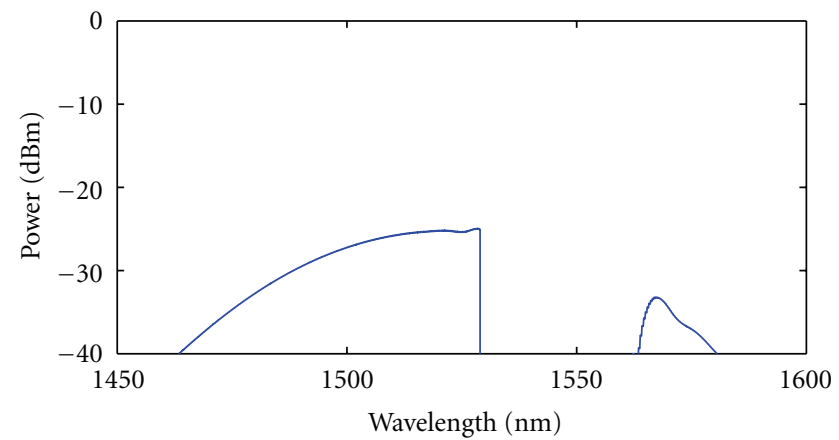

(a)

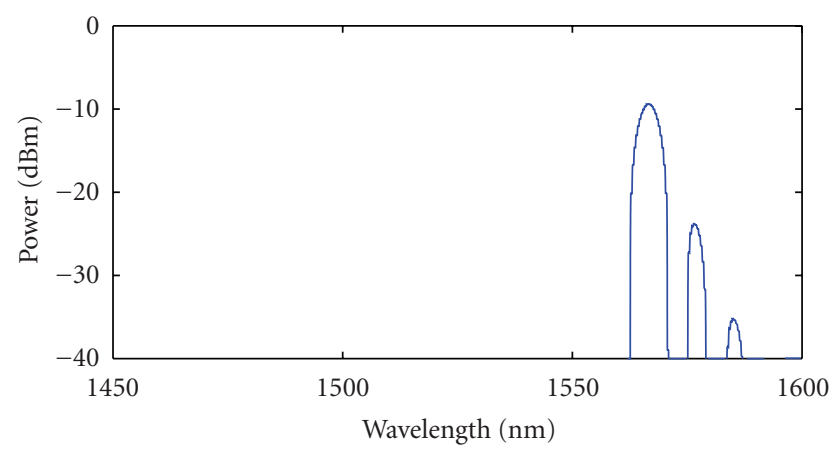

(c)

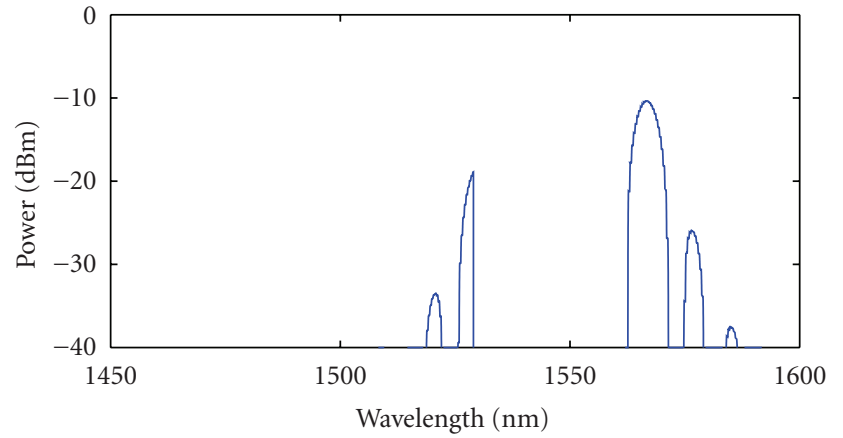

(b)

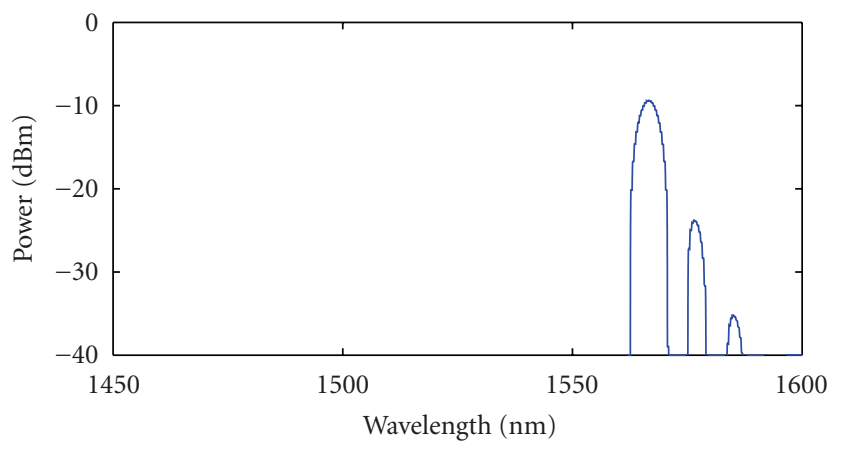

(d)

Figure 7: Modeled SOA2 ASE spectrum (counterclockwise). (a) Loop number = 1; (b) loop number = 10; (c) loop number = 20; $(\mathrm{d})$ loop number $=100$.

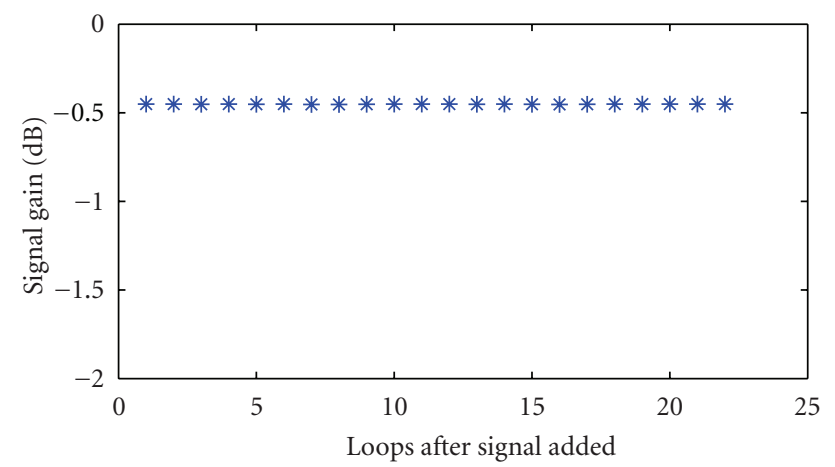

Figure 8: CW light $(1550 \mathrm{~nm},-20 \mathrm{dBm})$ gain as a function of circulating ASE loops after AGC-SOA stabilisation.

As ASE circulations progress, ASE emission in the S-band is further restrained becoming negligible, and ASE within the L-band accounts for gain clamping. This results from the fact that the lasing threshold is lower at longer wavelengths hence once lasing action is established, the shorter wavelength energy states are depleted. The results agree well with experimental observation.

Having established the steady-state conditions of model operation, an input optical signal was introduced after the ASE inside the AGC-SOA cavity stabilizes. The gain of $1550 \mathrm{~nm}$ CW light as a function of travelling ASE loop numbers is depicted in Figure 8. An optical signal power of $-20 \mathrm{dBm}$ was injected into SOA1. The drive current of SOA1 was set at $200 \mathrm{~mA}$, and SOA2 at $65 \mathrm{~mA}$. The simulation demonstrates that the signal gain settles within the first ASE loop transit and then remains unchanged; thus, after AGCSOA stabilizes from the initial state (shown in the evolution depicted in Figure 7), it takes $<2$ ns (within one loop time) for the gain to settle.

The variation of the gain with clamping SOA drive current was modelled over a range of clamping currents to corroborate that the model faithfully reproduced the experimental behaviour of the AGC-SOA. The model outputs (Figure 9) indicate broadly that the model is predicting the trends. There is no significant loss of $P_{\text {sat }}$ with gain reduction, however, the exact values of gain and $P_{\text {sat }}$ differ slightly from experimental measurements. This difference most likely derives from small differences between the physical parameters used in the model and the real device.

The DC parametric operation of the AGC-SOA can be estimated using the above model. CW light $(1550 \mathrm{~nm})$ was introduced into AGC-SOA once the steady-state operation was established. The input signal power was then increased steadily from $-35 \mathrm{dBm}$ to $20 \mathrm{dBm}$, and the normal performance metrics of gain, maximum output power at gain saturation $\left(P_{\text {sat }}\right)$, and noise figure $(\mathrm{NF})$ were recorded for a given clamping current condition; the clamping current is changed from $0 \mathrm{~mA}$ to $200 \mathrm{~mA}$. The simulation results are presented as a function of clamping current in Figure 10. The overall trends given by the models are in good agreement with previously reported experimental characterizations [6]. Gain clamping begins at a clamping bias current of greater than 


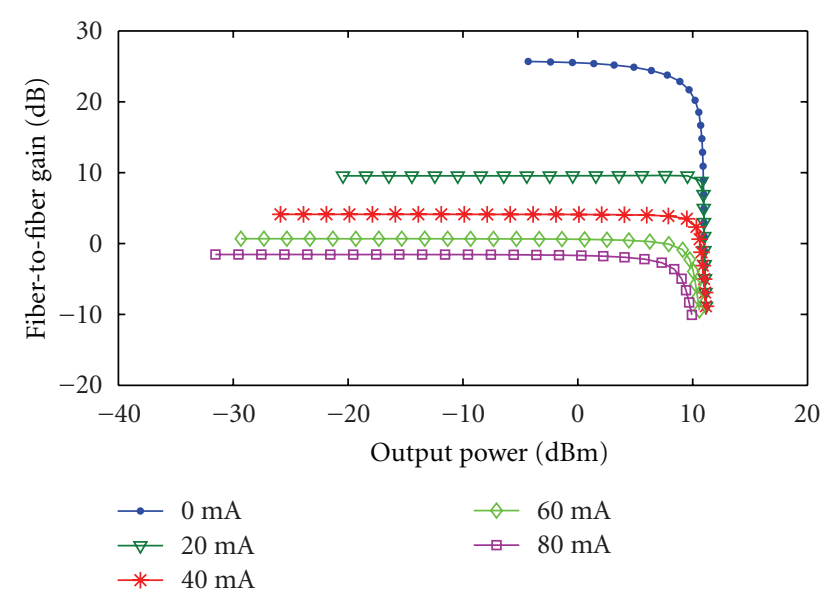

FIGURE 9: AGC-SOA gain as a function of output power at different clamping currents (modeling result).

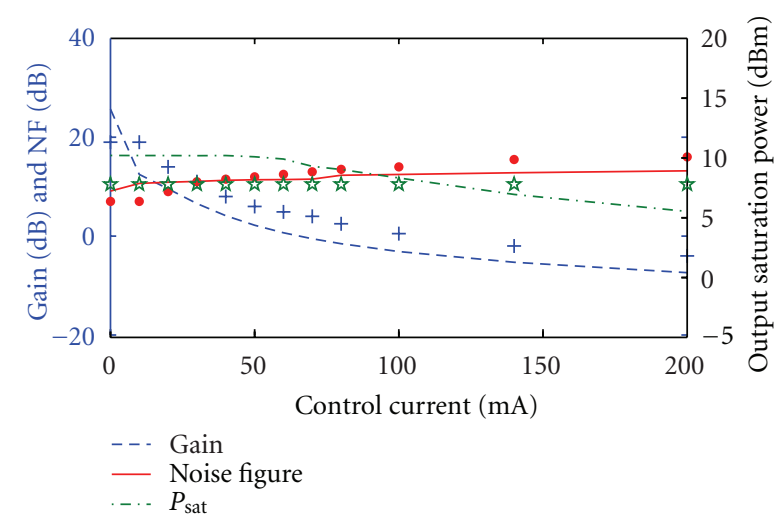

FIGURE 10: Experimental [6] and theoretical steady-state AGC-SOA performance comparisons. Red line: simulation results of NF. Red dot: experimental results of NF. Blue line: simulation results of gain. Blue cross: experimental results of gain. Green line: simulation results of $P_{\text {sat }}$. Green star: experimental results of $P_{\text {sat }}$.

$0 \mathrm{~mA}$. Experimentally this value was observed to be nearer $10 \mathrm{~mA}$ before there was sufficient gain within the clamping SOA to overcome loop losses and allow the lasing mode to stabilize. This difference is mainly due to the overestimation of ASE noise within the model [8]. Strong clamping is observed when the control SOA is operated at high gain levels; here, the AGC-SOA is driven into attenuation. As the gain is clamped, the NF increases, but in the main, the $P_{\text {sat }}$ value remains relatively constant when the clamping current is $<100 \mathrm{~mA}$.

The theoretical model could also be used to better understand the dynamic behaviour of the AGC-SOA. In order to do this, the model was run under the following conditions. Firstly, stable operation of the AGC-SOA was ensured by running the simulation with only ASE for the first 30 loop iterations and at an SOA2 (clamping SOA) bias of $20 \mathrm{~mA}$. At this point, a $0 \mathrm{dBm}$ input signal was introduced, and it can be seen that the gain of the AGC-SOA is around $10 \mathrm{~dB}$. The clamping SOA bias current was then increased every 10

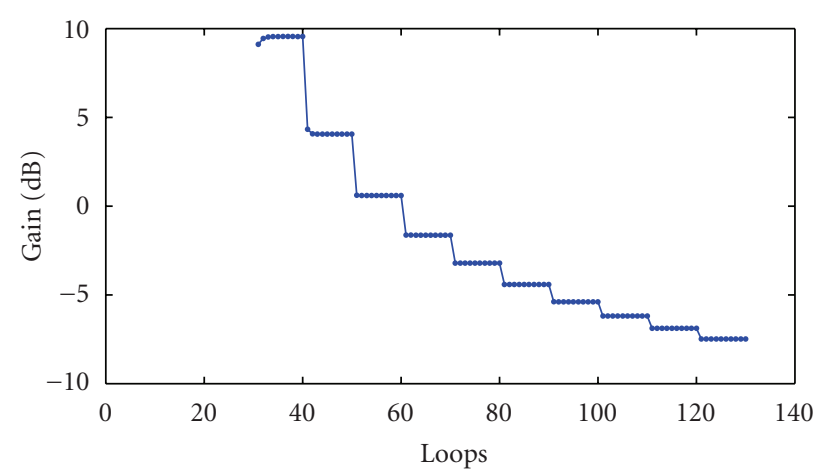

Figure 11: Dynamic gain variation as a function of clamping current.

loops, from $20 \mathrm{~mA}$ to $200 \mathrm{~mA}$, in steps of $20 \mathrm{~mA}$ and the gain change observed (Figure 11). It is clear from these simulations that the gain is indeed adjusted and stabilized within 1 or 2 loops of the model iteration. This implies therefore that the gain can be adjusted within nanosecond timescales which agree with experimental results [7].

\section{Conclusion}

A detailed wideband model for adjustable gain-clamped semiconductor optical amplifier (AGC-SOA) has been established, which can be used to analyse steady-state and dynamic gain modulation performance of the device. The simulations agree with the experimental results, showing the advantage of AGC-SOA not only lies in extended linear amplification regime and adjustable device gains, but also the ability of adjusting and stabilising its gain within nanosecond timescales. This feature could enable the device to work as power equalizer and linear amplifier in packet-based dynamic systems such as passive optical networks (PONs).

\section{Acknowledgments}

Strathclyde University gratefully acknowledges funds received from the British Council under the Prime Ministers Initiative II, Research Cooperation. This work was also supported in part by the Glasgow Research Partnership in Engineering (GRPe).

\section{References}

[1] L. Banchi, M. Presi, A. D’Errico, G. Contestabile, and E. Ciaramella, "All-optical wavelength 10 and 40 Gbit/s RZ-to-NRZ format and wavelength conversion using semiconductor optical amplifiers," Journal of Lightwave Technology, vol. 28, no. 1, pp. 32-38, 2010.

[2] A. Rostami, H. B. A. Nejad, R. M. Qartavol, and H. R. Saghai, "Tb/s optical logic gates based on quantum-dot semiconductor optical amplifiers," IEEE Journal of Quantum Electronics, vol. 46, no. 3, pp. 354-360, 2010. 
[3] C. Bohémond, A. Sharaiha, T. Rampone, and H. Khaleghi, "Electro-optical radiofrequency mixer based on semiconductor optical amplifier," Electronics Letters, vol. 47, no. 5, pp. 331333, 2011.

[4] P. Doussiere, F. Pommereau, J. Y. Emery et al., " $1550 \mathrm{~nm}$ polarization independent DBR gain clamped SOA with high dynamic input power range," in Proceedings of the 22nd European Conference on Optical Communication (ECOC '96), vol. 3, pp. 169-172, September 1996.

[5] D. A. Francis, S. P. DiJaili, and J. D. Walker, "A single-chip linear optical amplifier," in Proceedings of the Optical Fiber Communication Conference, pp. PD13/1-PD13/3, Anaheim, Calif, USA, March 2001.

[6] C. Michie, A. E. Kelly, I. Armstrong, I. Andonovic, and C. Tombling, "An adjustable gain-clamped semiconductor optical amplifier (AGC-SOA)," Journal of Lightwave Technology, vol. 25, no. 6, pp. 1466-1473, 2007.

[7] L. Liu, C. Michie, A. E. Kelly, and I. Andonovic, "Packet equalisation in PONs using adjustable gain-clamped semiconductor optical amplifiers (AGC-SOA)," in Proceedings of the International Conference on Transparent Optical Networks (ICTON '11), pp. 1-4, Stockholm, Sweden, June 2011.

[8] M. J. Connelly, "Wideband semiconductor optical amplifier steady-state numerical model," IEEE Journal of Quantum Electronics, vol. 37, no. 3, pp. 439-447, 2001.

[9] ITU-T Recommendation G.987.2, “10-Gigabit-capable passive optical networks (XG-PON): physical media dependent (PMD) layer specification," January 2010.

[10] X. H. Jia, "Theoretical investigation of gain-clamped semiconductor optical amplifiers using the amplified spontaneous emission compensating effect," Journal of the Optical Society of America B, vol. 23, no. 12, pp. 2503-2510, 2006.

[11] A. Matsumoto, K. Nishimura, K. Utaka, and M. Usami, “Operational design on high-speed semiconductor optical amplifier with assist light for application to wavelength converters using cross-phase modulation," IEEE Journal of Quantum Electronics, vol. 42, no. 3, Article ID 01597418, pp. 313-323, 2006.

[12] C. Y. Jin, Y. Z. Huang, L. J. Yu, and S. L. Deng, "Detailed model and investigation of gain saturation and carrier spatial hole burning for a semiconductor optical amplifier with gain clamping by a vertical laser field," IEEE Journal of Quantum Electronics, vol. 40, no. 5, pp. 513-518, 2004.

[13] S. Verspurten, G. Morthier, and R. Baets, "Experimental and numerical small-signal analysis of two types of gain-clamped semiconductor optical amplifiers," IEEE Journal of Quantum Electronics, vol. 42, no. 3, Article ID 01597417, pp. 302-312, 2006.

[14] S. L. Chuang, Physics of Optoelectronic Devices, Wiley-Interscience, New York, NY, USA, 1995.

[15] M. J. Connelly, "Wide-band steady-state numerical model and parameter extraction of a tensile-strained bulk semiconductor optical amplifier," IEEE Journal of Quantum Electronics, vol. 43, no. 1, pp. 47-56, 2007.

[16] A. Yariv, Optical Electronics in Modern Communications, Oxford University Press, New York, NY, USA, 5th edition, 1997. 

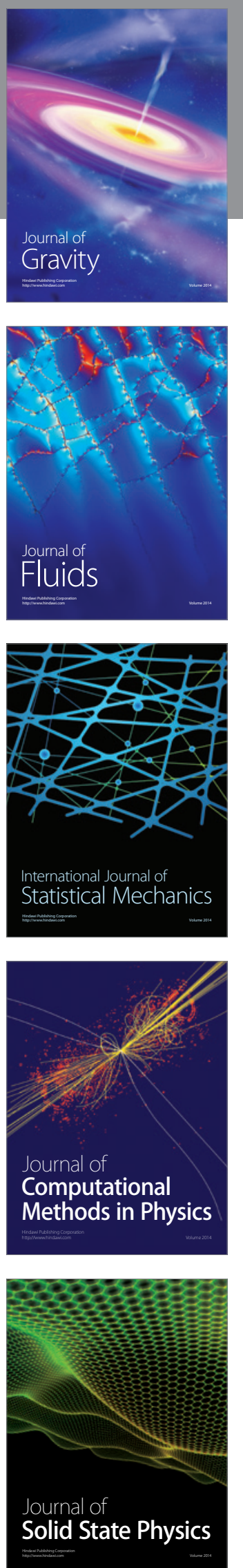

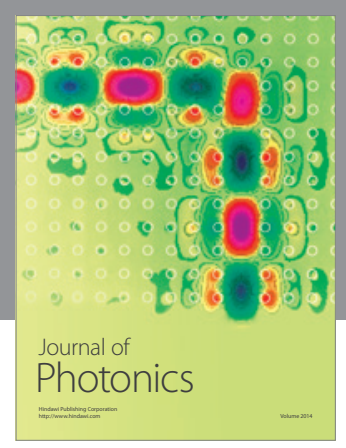

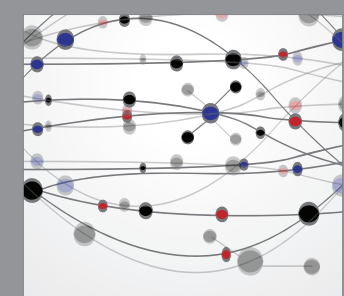

The Scientific World Journal
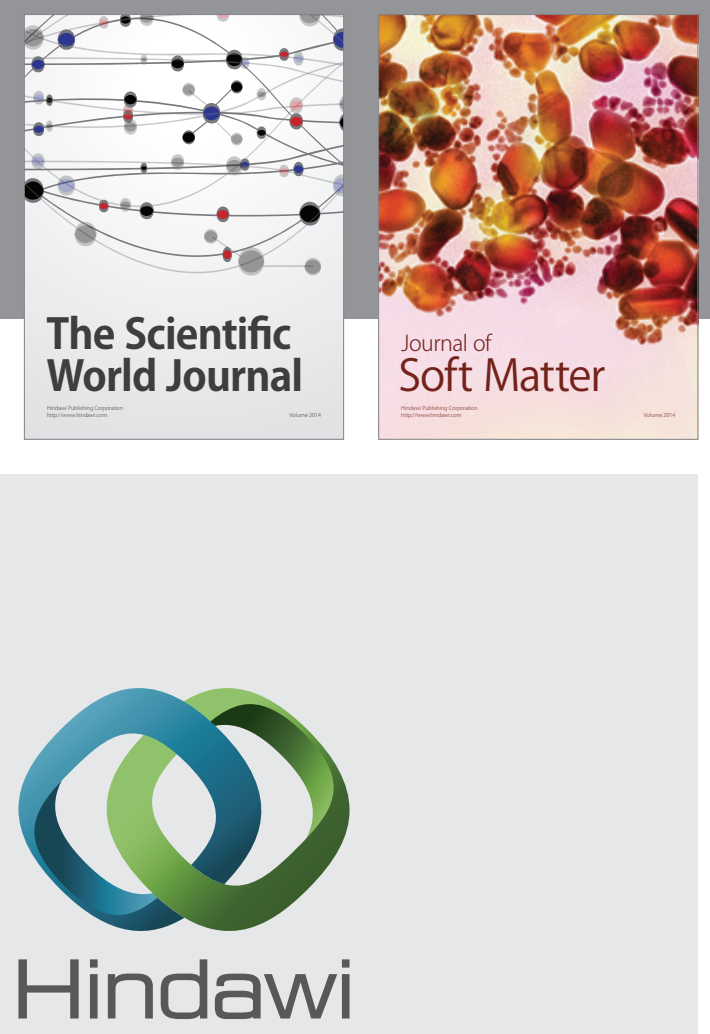

Submit your manuscripts at

http://www.hindawi.com
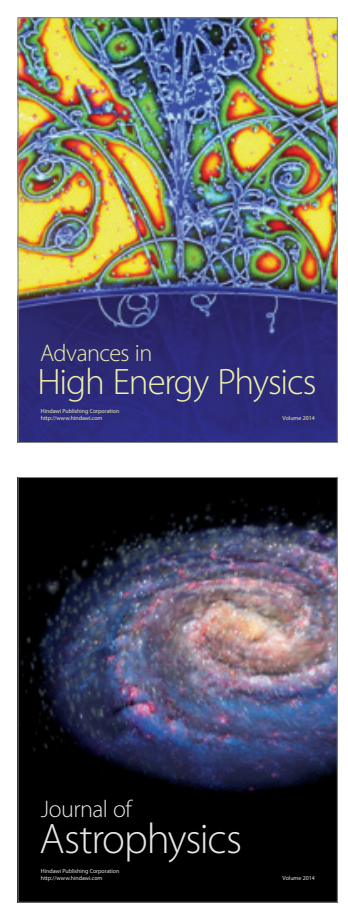
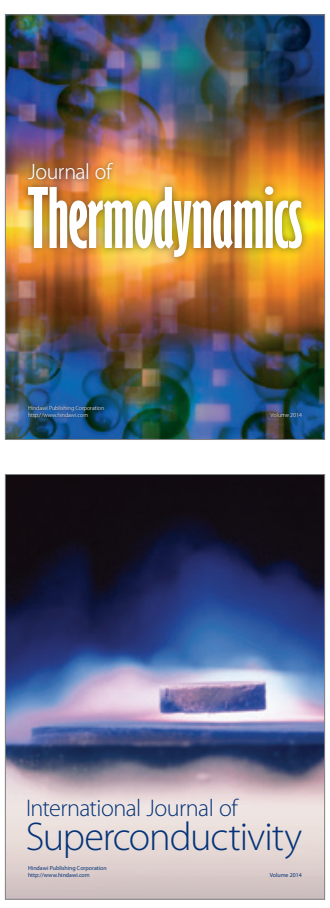
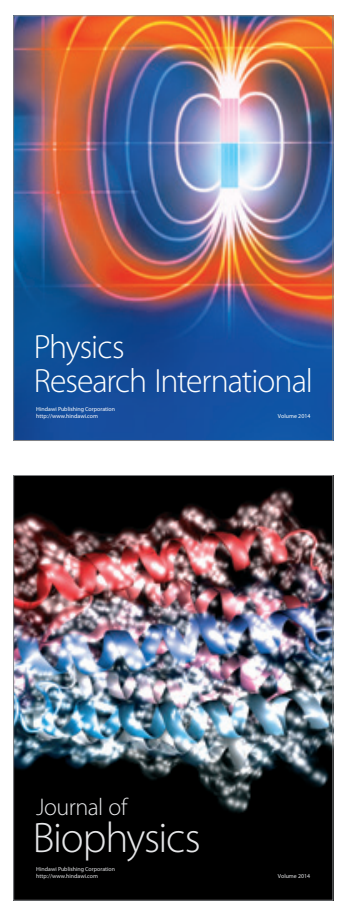
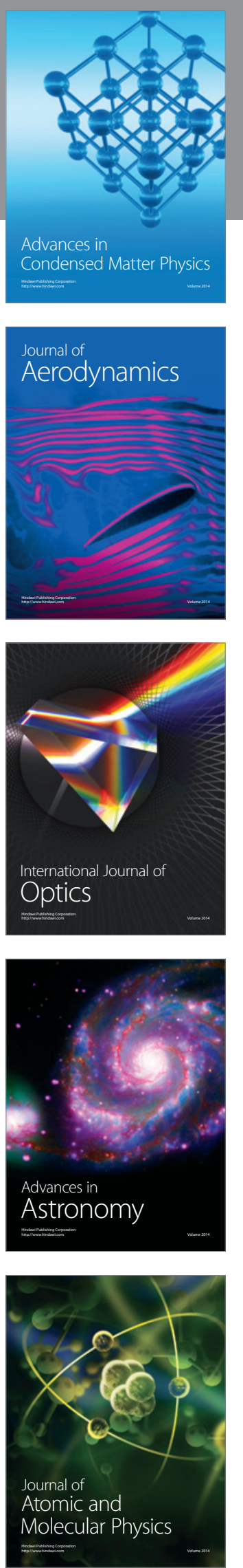